\title{
Subcortical and Neocortical Mechanisms of Visual-Somatosensory Interplay
}

\author{
Flavia Mancini \\ Department of Neuroscience, Physiology, and Pharmacology, Institute of Cognitive Neuroscience, University College London, London, WC1N 3AR, United \\ Kingdom \\ Review of Sieben et al.
}

The concept of unisensory cortices has been challenged by growing evidence that neurons in primary sensory regions respond to stimuli of different modalities (Driver and Noesselt, 2008). The traditional view is that these crossmodal influences result from backward projections from higher-order areas to primary cortical areas. This feedback hypothesis is supported by a large body of evidence showing crossmodal attentional modulation in lowerorder sensory regions (Driver and Noesselt, 2008). More recently, it has been proposed that feedforward and lateral pathways at the earliest processing stages also mediate multisensory interactions. This view is based on two lines of evidence. First, multisensory interactions in sensory cortices first appear 30-50 ms after stimulus onset. Thus, they are faster than those resulting from feedback connections. Second, anatomical studies reveal direct connections between primary sensory cortices in nonprimate animals (Schroeder and Foxe, 2005). The impact of lateral cortical connectivity on multisensory interactions is unclear.

A recent report by Sieben et al. (2013) provided anatomical and electrophysiological evidence for lower-order inter-

Received May 24, 2013; revised June 27, 2013; accepted July 1, 2013.

F.M. is supported by a Wellcome Trust Project Grant 094863/Z/10/Z

Correspondence should be addressed to Flavia Mancini, Department of Neuroscience, Physiology, and Pharmacology, Institute of Cognitive Neuroscience, University College London, 17 Queen Square, London, WC1N 3AR, United Kingdom. E-mail: f.mancini@ucl.ac.uk.

DOI:10.1523/JNEUROSCI.2222-13.2013

Copyright $\odot 2013$ the authors $\quad 0270-6474 / 13 / 3312555-02 \$ 15.00 / 0$ actions between primary visual and somatosensory cortex (V1 and S1). The authors extracellularly recorded electrical activity evoked by unimodal visual (light flashes) or tactile stimulation (whisker deflection) from multiple sites in V1 and S1. Unimodal stimulation was compared with bimodal stimulation consisting of flash and whisker deflection simultaneously presented in the same (congruent) or in opposite (incongruent) hemifields. To investigate whether the visual cortex mediates visual-somatosensory interactions, the authors blocked action potentials in V1 by intracortical injection of lidocaine, and studied the effect of this block on somatosensory evoked potentials, as well as stimulus-induced and spontaneous oscillations in S1. Finally, anatomical tracing of neurons in S1 and V1 was performed postmortem.

Extracellular recordings from all layers of the barrel field in S1 revealed that bimodal stimulation enhanced the early components of evoked potentials, compared with unimodal stimulation. In the rat, evoked potentials are characterized by a first large positive component (P1), followed within $10 \mathrm{~ms}$ by a negative component (N1), and then by two slower components (N2 and P2). Congruent bimodal stimulation elicited a superadditive augmentation of the P1 and N1 components of evoked potentials originating in the granular layers of S1.

Sieben et al. (2013) suggest that subcortical feedforward pathways that bypass
V1 mediate the cross-modal augmentation of early evoked responses in S1. Their interpretation is based on two findings. First, the onset of the first peak of bimodal enhanced potentials in S1 had a shorter latency than the onset of visual evoked responses in V1. Second, lidocaine-induced blockade of $\mathrm{V} 1$ activity did not affect the bimodal augmentation of the first peak (P1), while eliminating the enhancement of the second peak (N1). Indeed, the N1 is often thought to reflect corticocortical interactions (Szentagothai, 1978).

Sieben et al. (2013) also investigated the effect of vision on both spontaneous and induced somatosensory oscillations. Unimodal visual stimulation reset the phase of $\theta$ and $\alpha$ spontaneous oscillations bilaterally in all layers of S1, but most prominently in the granular layers of the contralateral barrel field. Similar phase reset of slow oscillations was also observed in V1 after unimodal tactile stimulation. Furthermore, visual input modulated stimulus-induced somatosensory oscillations. Lidocaine-induced blockade of V1 both decreased and delayed the phase concentration of $\Theta$ and $\alpha$ band oscillations by contralateral visual input. In addition, silencing V1 eliminated most of the power differences between bimodal and unimodal stimulus-induced responses.

Finally, anatomical tracing revealed sparse, direct, intrahemispheric connectivity between the granular and supragranular layers of V1/V2 and S1. Sieben et al. (2013) propose that this corticocortical 
connectivity accounts for visual modulation of both spontaneous and induced somatosensory oscillations.

Overall, the authors conclude that visual and tactile inputs are integrated at both subcortical and neocortical levels, supporting the view that feedforward and lateral connections contribute to multisensory processing. More specifically, they suggest that thalamic integration is responsible for supraadditive augmentation of somatosensory evoked responses, while direct projections from V1 modulate the power of induced network oscillations in $\mathrm{S} 1$ by resetting their phase.

The effects of blocking V1 activity point to a role for this region in the modulation of somatosensory activity, and the finding of direct connectivity between V1 and $\mathrm{S} 1$ is also suggestive of direct communication between these regions. However, the evidence in support of the hypothesis that direct connectivity between S1 and V1 mediates changes in somatosensory oscillations is not conclusive. Indeed, it cannot be excluded that V1 modulates S1 activity indirectly, through higher-order convergence areas.

The ultimate challenge is to understand the specific role of direct connectivity between sensory cortices in multisensory processing and behavior. A recent study on visual-auditory interactions demonstrated that activation of primary auditory cortex (A1) by a noise burst suppresses visually driven behavioral responses, by driving local GABAergic inhibition on V1 via corticocortical connections (Iurilli et al., 2012). The role of V1/A1 connectivity was demonstrated through transection of these connections. This evidence thus supports the view that corticocortical connectivity mediates multisensory interactions.

In humans, there is extensive evidence of cross-modal modulation of activity in primary sensory cortices (Ghazanfar and Schroeder, 2006; Driver and Noesselt, 2008). Given the pervasiveness of multisensory interactions at the early stages of sensory processing, it has been proposed that the neocortex is essentially multisensory (Ghazanfar and Schroeder, 2006). This view finds novel support in a functional magnetic imaging (fMRI) study using multivariate pattern analysis in humans. Liang et al. (2013) demonstrated that unimodal stimulation (visual, tactile, auditory, or nociceptive) elicits distinguishable spatial patterns of neuronal responses in both their corresponding primary sensory cortex, and in other primary sensory cortices. It is, however, unclear whether feedback, feedforward, or lateral connections drive multisensory responses at neocortical level.

Interestingly, the study by Sieben et al. (2013) indicates the phase reset of network oscillations as a possible mechanism of multisensory interaction. Similar phase resetting has been demonstrated in monkeys as a correlate of integration between auditory and somatosensory inputs (Lakatos et al., 2007), and it is thought to facilitate sensory processing. However, the effect on behavior has not been investigated in the present study.

Finally, it would be important to understand whether the mechanisms of visual-somatosensory interplay described by Sieben et al. (2013) are specific for tactile input, or may extend also to nociceptive signals. For example, future studies could determine whether V1-S1 connections target a specific area in S1, and whether they make synapses on specific cell types. Although nociceptive inputs are strongly modulated by other sensory inputs (Mancini et al., 2012), the underlying neural correlates of these interactions are rarely investigated. In humans, they may involve a change in the functional coupling between visual and somatosensory areas (Longo et al., 2012), possibly dependent on excitability shifts in the occipital cortex (Mancini et al., 2012).

In conclusion, the strength of the work by Sieben et al. (2013) is to combine an array of electrophysiological measures with pharmacological interventions and anatomical tracing. Their results are suggestive of subcortical and neocortical mechanisms of visual-somatosensory interplay. The hope is that their valuable work will stimulate research oriented at understanding the specific contribution of corticocortical connectivity to multisensory processing and perception.

\section{References}

Driver J, Noesselt T (2008) Multisensory interplay reveals crossmodal influences on 'sensoryspecific' brain regions, neural responses, and judgments. Neuron 57:11-23. CrossRef Medline

Ghazanfar AA, Schroeder CE (2006) Is neocortex essentially multisensory? Trends Cogn Sci 10:278-285. CrossRef Medline

Iurilli G, Ghezzi D, Olcese U, Lassi G, Nazzaro C, Tonini R, Tucci V, Benfenati F, Medini P (2012) Sound-driven synaptic inhibition in primary visual cortex. Neuron 73:814-828. CrossRef Medline

Lakatos P, Chen CM, O'Connell MN, Mills A, Schroeder CE (2007) Neuronal oscillations and multisensory interaction in primary auditory cortex. Neuron 53:279-292. CrossRef Medline

Liang M, Mouraux A, Hu L, Iannetti GD (2013) Primary sensory cortices contain distinguishable spatial patterns of activity for each sense. Nat Commun 4:1979. CrossRef Medline

Longo MR, Iannetti GD, Mancini F, Driver J, Haggard P (2012) Linking pain and the body: neural correlates of visually induced analgesia. J Neurosci 32:2601-2607. CrossRef Medline

Mancini F, Bolognini N, Haggard P, Vallar G (2012) tDCS modulation of visually induced analgesia. J Cogn Neurosci 24:2419-2427. CrossRef Medline

Schroeder CE, Foxe J (2005) Multisensory contributions to low-level, 'unisensory' processing. Curr Opin Neurobiol 15:454-458. CrossRef Medline

Sieben K, Röder B, Hanganu-Opatz IL (2013) Oscillatory entrainment of primary somatosensory cortex encodes visual control of tactile processing. J Neurosci 33:5736-5749. CrossRef Medline

Szentágothai J (1978) The Ferrier Lecture, 1977. The neuron network of the cerebral cortex: a functional interpretation. Proc R Soc Lond B Biol Sci 201:219-248. CrossRef Medline 